CAHIER 2097

\title{
THE MACROECONOMIC EFFECTS OF INFREQUENT INFORMATION WITH ADJUSTMENT COSTS
}

\author{
Marco BONOMO' and René GARCIA ${ }^{2}$ \\ $1 \quad$ Pontificia Universidade Católica do Rio de Janeiro \\ 2 Centre de recherche et développement en économique (C.R.D.E.) and \\ Département de sciences économiques, Université de Montréal, and CIRANO
}

October 1997

\begin{abstract}
We would like to thank Eduardo Engel and Steve Ambler for their comments, and Carlos Viana de Carvalho and Benoit Brillon for their excellent research assistance. Financial support by the PARADI Research Program funded by the Canadian International Development Agency (CIDA) is gratefully acknowledged. The first author would also like to thank CNPq (National Research Council of Brazil) for financial support. The second author thanks the Fonds pour la formation de chercheurs et l'aide à la recherche (FCAR) of Québec for financial assistance.
\end{abstract}




\section{RÉSUMÉ}

$\mathrm{Au}$ cours de la dernière décennie, plusieurs articles on étudié les effets macroéconomiques que pouvaient entraîner des ajustements importants et intermittents de variables de décision microéconomiques telles que les prix, les investissements, la consommation de biens durables ou l'emploi, un comportement qui peut se justifier par la présence de coudes dans les fonctions de coûts d'ajustement. Dans ces modèles, les agents observent continuellement le niveau optimal de leur variable de décision. Dans le présent article, nous développons un modèle similaire mais où justement les agents n'observent pas continuellement ce niveau optimal car l'information arrive par intermittence comme dans le cas des publications de statistiques économiques ou des annonces de dividendes. Nous trouvons d'abord la règle optimale de décision individuelle, qui dépend à la fois du temps et de l'état de la nature. Nous développons ensuite un cadre d'agrégation afin d'étudier les implications macroéconomiques de ces règles optimales de décision individuelle. Une caractéristique distinctive de notre modèle est qu'un grand nombre d'agents tendent à agir en même temps, et ce, d'autant plus que l'incertitude est importante. L'effet moyen d'un choc agrégé est en relation inverse avec l'amplitude du choc et l'incertitude agrégée. Nous montrons que ces effets sont nettement différents des effets obtenus avec des modèles à coûts d'ajustement avec information complète.

Mots clés: effets macroéconomiques d'information intermittente, coûts d'ajustement, règles de décision optimales avec coûts d'ajustement

\section{ABSTRACT}

In the last decade, the potential macroeconomic effects of intermittent large adjustments in microeconomic decision variables such as prices, investment, consumption of durables or employment - a behavior which may be justified by the presence of kinked adjustment costs - have been studied in models where economic agents continuously observe the optimal level of their decision variable. In this paper, we develop a simple model which introduces infrequent information in a kinked adjustment cost model by assuming that agents do not observe continuously the frictionless optimal level of the control variable. Periodic releases of macroeconomic statistics or dividend announcements are examples of such infrequent information arrivals. We first solve for the optimal individual decision rule, that is found to be both state and time dependent. We then develop an aggregation framework to study the macroeconomic implications of such optimal individual decision rules. Our model has the distinct characteristic that a vast number of agents tend to act together, and more so when uncertainty is large. The average effect of an aggregate shock is inversely related to its size and to aggregate uncertainty. We show that these results differ substantially from the ones obtained with full information adjustment cost models.

Key words : macroeconomic effects of infrequent information, adjustment costs, state dependent and time dependent rules 


\section{Introduction}

In the last decade, the macroeconomic literature paid considerable attention to the potential aggregate effects of intermittent large adjustments in microeconomic decision variables such as prices (Caplin and Spulber, 1987, Caplin and Leahy, 1991, Caballero and Engel, 1993 and 1992), investment (Caballero and Engel, 1994), inventories (Caplin, 1985), consumption of durables (Caballero, 1993) or employment (Caballero, Engel and Haltiwanger, 1994). A distinctive feature of this literature is that explicit aggregation of individual rules is undertaken, resulting in rich dynamic patterns for the aggregate variables which are in sharp contrast with the inertial behavior at the microeconomic level. In these adjustment cost models, economic agents always observes the frictionless optimal level of the control variable and infrequent adjustments may be justified by optimal behavior in the presence of kinked adjustment costs (Bertola and Caballero, 1990) ${ }^{1}$.

In this paper we develop a simple model which introduces imperfect information in a kinked adjustment cost model by assuming that agents do not observe continuously the frictionless optimal value of the control variable. This infrequent flow of information can be considered either as exogenous or endogenous to the agent's decision. Examples of exogenous flows are intermittent information arrivals: macroeconomic statistics such as inflation, level of employment or GNP are published periodically, dividends of firms are announced only at certain dates, markets close regularly on weekdays and holidays. In all these cases, agents do not observe continuously the variable of interest. Such an intermittent information arrival has the interesting implication that a large number of agents receive the same information at the same time, creating the conditions for a potential mass reaction. Indeed, increased volatility of financial markets around dividend announcements and macroeconomic data releases have been documented in numerous articles ${ }^{2}$. Infrequent information could also result from the optimal decision of the agent to gather information in the presence of information collection costs. This endogenous

\footnotetext{
${ }^{1}$ When the adjustment cost function has a kink at the point of no-adjustment, it is best for the agent not to adjust for small changes of the frictionless optimal level. Adjustment is triggered when the discrepancy between the control variable and its optimal level becomes large enough.

${ }^{2}$ See Cornell (1978), for dividend announcements and Harvey and Huang $(1991,1992)$, Ederington and Lee (1993), for macroeconomic data releases.
} 
infrequent gathering would not necessarily coordinate agents' reactions and will have different macroeconomic implications. In this paper, we focus our attention on the first source of infrequent information, i.e. the exogenous arrival of macroeconomic information for all agents at the same time.

Our model has the distinct characteristic that a vast number of agents tend to act together, and more so when uncertainty is large ${ }^{3}$. We show that lump-sum adjustment costs interact with infrequent information to generate effects of aggregate shocks on macroeconomic variables that differ substantially from the ones obtained with continuous information adjustment cost models. First, the relative effect of cumulative aggregate shocks decreases sharply with the size of the shock. Second, the relative average effect of these shocks decreases when aggregate uncertainty increases. Other results are more similar in both models. When idiosyncratic uncertainty increases, the average effect decreases, while an increase in the adjustment cost raises the average effect.

In order to perform the comparisons above, we solve the microeconomic problem of finding the optimal policy in the presence of both lump-sum adjustment costs and infrequent information about the value of the frictionless optimal level of the control variable. To make the conditions which determine the optimal policy as simple as possible while keeping the main insights of the model, we assume that the stochastic process of the frictionless optimal value of the control variable has no drift. We find that the optimal rule is for agents to adjust or not depending on the state at times of information arrivals. ${ }^{4}$ Therefore, it is both state and time dependent. Such a rule was conjectured by Blanchard and Fischer (1989, p. 413) as the rule that could result from a combination of infrequent information about the state variable and adjustment costs. The difference with our model is that they justify the infrequent gathering of information by the existence of costs of collecting

\footnotetext{
${ }^{3}$ Other authors have explained these mass reactions by different information extraction mechanisms. Banerjee (1992) proposes a model where individuals tend to act simultaneously, even when their private information would not bring by itself such coordination. Caplin and Leahy (1994) provide a rationale for market collapses or crashes based on a discontinuous evolution of public information, which results from difficulties in aggregating private information.

${ }^{4}$ The presence of a large drift will make it optimal for agents to adjust between information collections. The conditions determining the optimal policy in the presence of a drift are quite complex.
} 
information.

The optimal rule in this case is characterized by a single parameter $s$, which determines the inaction range $(-s, s)$ for the discrepancy between the frictionless optimal value of the control variable and its actual value, at times of information arrival. We show that the inaction barriers are much tighter than in the continuous information model. When the adjustment cost is sufficiently low, the barriers are quite insensitive to the uncertainty governing the stochastic process assumed for the optimal level of the control variable. On the other hand, an increase in the adjustment cost brings about a relatively larger increase in the barriers when information is infrequent than when it is continuous.

Ball and Mankiw (1994) also explore the consequences of a price rule that is both time and state dependent. The agents adjust without paying a menu cost at even periods. Adjustments at odd periods will be made only if the benefit of doing so is greater than the menu cost. The frictionless optimal price is always known. They focus mainly on the effect of the drift in the frictionless optimal price process on output dynamics. In our model, we assume the drift to be zero and adjustment costs are always present. Our main goal is to illustrate the interaction between adjustment costs and infrequent information.

The rest of the paper is organized as follows. In Section 2, we derive the optimal rule in the presence of both lump-sum adjustment costs and infrequent observation of the optimal level of the control variable. Section 3 evaluates the aggregate effect of macroeconomic shocks through the optimal adjustment of microeconomic units. Concluding remarks are presented in Section 4 .

\section{The Optimal Rule}

In this section, we set up the optimization problem of the agents confronted with infrequent information and adjustment costs, derive the optimal decision rule, and investigate the implications of this rule for various configurations of adjustment costs and uncertainty and in relation with the continuous information case. 


\subsection{Assumptions and formulation of the optimization problem}

An agent faces the problem of setting optimally the level of a control variable $x$, be it price, employment or investment for a firm, or consumption of some durable good for a household, in the presence of two types of costs: a lumpsum adjustment cost, $k$, when resetting, and an instantaneous flow cost when its control variable drifts away from a frictionless optimal level, $x^{*}$. For simplicity, we will assume a quadratic form $\left(x-x^{*}\right)^{2}$ for the latter cost ${ }^{5}$. Time is discounted by a constant instantaneous discount rate $\rho$. We depart from the previous literature by assuming that information about the optimal level $x^{*}$ arrives at discrete time intervals ${ }^{6}$. Although the agent does not observe $x^{*}$ between two successive information arrivals, he can form probabilistic assessments about the value of $x^{*}$ given his information, which consists of the past observations of $x^{*}$ at the discrete information times. We assume, again for simplicity, that $x^{*}$ is a driftless Brownian motion with diffusion parameter $\sigma$, i.e.:

$$
d x_{t}^{*}=\sigma d w_{t}
$$

where $w$ is a Wiener process. ${ }^{7}$

The distribution of $x_{t}^{*}$, conditional on past observations of $x^{*}$ at the discrete information times, depends only on the last observation $x_{u}^{*}$, where $u$ is the time of the last information arrival. The distribution of $x_{t}^{*}$ conditioned on the knowledge of $x_{u}^{*}$, for $u<t$, is normal with zero mean and variance $\sigma^{2}(t-u)$.

Given initial values for the control variable and the frictionless optimal level, the agent minimizes the expected present value of both the adjustment cost and the flow cost of deviating from the frictionless optimal level of the control variable. The expected value, at the time of the last information

\footnotetext{
${ }^{5}$ Quadratic flow costs could be justified as a second-order approximation to the loss in profit or utility caused by a non-optimal level of the control variable.

${ }^{6}$ An excellent exposition of optimal control problems under adjustment costs when the frictionless optimal value of the control variable is always known is found in Dixit (1993).

${ }^{7}$ The assumption of an exogenous process for $x^{*}$ is unrealistic in many settings, but the difficulty of modeling $x^{*}$ with an endogenous component as well as the specificity of this modeling to the particular setting considered prevented us from pursuing such a strategy. We believe that the main insights derived from the model will remain even if the $x^{*}$ process is partly endogenous.
} 
arrival $u$, of the flow cost at time $\mathrm{t}$ is $E_{u}\left(x_{t}-x_{t}^{*}\right)^{2}$ and can be decomposed as follows:

$$
E_{u}\left(x_{t}-x_{t}^{*}\right)^{2}=\left(x_{t}-E_{u} x_{t}^{*}\right)^{2}+E_{u}\left(x_{t}^{*}-E_{u} x_{t}^{*}\right)^{2}
$$

The second term represents the irreducible cost of not being informed about the optimal value of the frictionless optimal value $x_{t}^{*}$. If there were no adjustment costs, the agent will minimize the expected quadratic flow costs by setting $x_{t}$ equal to $E_{u} x_{t}^{*}$. Since $x^{*}$ is driftless, it is a martingale, and $E_{u} x_{t}^{*}=x_{u}^{*}$, the value of the optimal variable when the last information arrived. Therefore, even in the absence of adjustment costs, there will be no adjustment between information arrivals ${ }^{8}$.

From the structure of the problem and from the Markovian nature of the stochastic process for $x^{*}$, it is clear that, given a discrepancy $x_{u}-x_{u}^{*}$ at the time of information arrival $u$, the value of the minimized cost starting at $u$ will be identical to the value at $u+n$ ( $\mathrm{n}$ being an integer) if the discrepancy is the same at that time. The discrepancy $x-x^{*}$ is therefore a sufficient state variable for the value function at times of information arrival. Since there will never be an adjustment between information arrivals, it suffices to consider the value function just at times following information arrivals.

\subsection{Solving the Optimization Problem}

It is never worthwhile to correct small deviations from the optimal level of the control variable because adjustment costs are lump-sum. Also, as the adjustment costs incurred depend neither on the state before adjusting nor on the size of the adjustment, the agent always adjusts to the same level of discrepancy $\left(x-x^{*}\right)$, which is zero in the case of a driftless process. Given the quadratic nature of the flow costs incurred by departing from the frictionless optimal value, the discrepancies which trigger an upward adjustment and a downward adjustment are symmetric around zero. Thus,

\footnotetext{
${ }^{8}$ When there are adjustment costs, if an adjustment takes place at the time of an information arrival, then it is obvious that there will be no adjustments before the next information arrival. It is not as obvious, although it is fortunately true, that when there is not adjustment when information arrives, there will be no adjustments before the next information arrival either. Thus, the assumption of no drift simplifies the problem enormously. When there is a drift, it is necessary to determine whether to adjust and the size of adjustments at all times.
} 
the optimal control policy is not to adjust between information arrival times, and to reset discrepancy to zero just after information arrival if its absolute level is greater or equal to a given value, which we call $s$. This rule is clearly time and state dependent. The value of $s$ is what remains to be determined.

We can determine it by solving a discrete time stochastic dynamic programming problem ${ }^{9}$, where at each time of information arrival, the agent decides either to pay the adjustment cost and to adjust the discrepancy to zero, and consequently $x$ to $x^{*}$, or to wait until the next period of information arrival. Without loss of generality, we set the length of the interval between information arrivals to one. Formally,

$$
V(y)=\min \left\{B(y)+e^{-\rho} E_{t} V(y-\epsilon), k+B(0)+e^{-\rho} E_{t} V(-\epsilon)\right\}
$$

where the function $B$ represents the expected discounted cost of departing from the frictionless optimal level of the control variable between now and the time of the next information arrival and $\epsilon$ is the shock to the frictionless optimal process between $t$ and $t+1$, which is a normal variable with zero mean and variance $\sigma^{2}$. The expression for $B$, derived in Appendix $A$, is:

$$
B(y)=\frac{y^{2}\left(1-e^{-\rho}\right)}{\rho}-\frac{\sigma^{2} e^{-\rho}}{\rho}+\frac{\sigma^{2}\left(1-e^{-\rho}\right)}{\rho^{2}}
$$

Equation 2 is valid for every $y$. The right-hand side can be viewed as a transformation $T$ of the function $V$. The right $V$ is found when $T(V)=V$. Because $T$ is a contraction mapping, $T^{n}(V)$ tends to $V$ as $n$ becomes large. Therefore, $V$ can be found by guessing an initial value for $V$ and interating until convergence. After the value function is found, the optimal policy can be evaluated.

Since the second argument of the min function does not depend on $y$ and, it can be stablished that, the first argument, and the value function itself, is increasing in $|y|$, there exists a cutoff discrepancy such that, below it, it is optimal not to adjust and, above it, adjustment is optimal. The level $s$ is therefore the discrepancy that makes the agent indifferent between adjusting or not adjusting:

\footnotetext{
${ }^{9}$ A nice introduction to discrete time stochastic dynamic programming is Sargent (1987), chapters 1 and 2.
} 


$$
B(s)+e^{-\rho} E_{t} V(s-\epsilon)=k+B(0)+e^{-\rho} E_{t} V(-\epsilon)
$$

When the discrepancy is zero, it is clearly optimal not to adjust, and the right hand side of the equation is equal to $V(0)+k$. As $s$ is the point that makes the agent indifferent between adjusting or not adjusting, the left hand-side is equal to the value function evaluated at $s$. Thus, the condition above can be restated as a value matching condition, which is a familiar one in the problem of optimal control with full information.

$$
V(s)=V(0)+k
$$

This condition must be satisfied both at $s$ and at $-s$, since the value function is symmetric. Then, once the value function is obtained, this equation can be used to determine $s$.

Note that with infrequent information, the value matching condition plays a different role than in the full information resetting problems. In the latter, the value matching condition is a condition of consistency which is always satisfied by the value function at the resetting and trigger points, even if these points are not optimal. In our problem, it is truly an optimality condition in the sense that it is only satisfied if an optimal $s$ is chosen. In other words, if we chose a non-optimal resetting point and calculated the value function for this resetting policy, there would be a discontinuity in the value function at such chosen resetting point.

\subsection{Numerical Results}

Table 1 reports the optimal rule parameter $s$ found numerically ${ }^{10}$ for different diffusion parameters of the frictionless optimal control process. For purposes of comparison, we also report the parameter value for the optimal rule when the agent has continuous information about the optimal value of the control variable. The optimal rule in this case is also symmetric and two-sided ${ }^{11}$, but

\footnotetext{
${ }^{10}$ The value function is computed numerically using a picewise linear approximation along a grid with a large number of points. We start with some initial value for this set of points and iterate until convergence. Once the value function is obtained, the s value is found by using condition (3).

${ }^{11}$ To compute the optimal s for the continuous information case, we use the formula in Bonomo (1992) for the two-sided optimal rule.
} 
adjustment occurs whenever the absolute value of the discrepancy is equal to the barrier.

The first pattern to notice is that the infrequent information bands are much narrower than the continuous information ones. Since the cost of departing from the optimal level of the control variable is a convex function of the discrepancy, infrequent monitoring has to be compensated with a stricter control when monitoring occurs.

The second pattern is that the size of the bands is much less sensitive to the variability of the frictionless optimal process in the infrequent information case. To build one's intuition, let us first note that changing the size of the band entails a trade-off. An increase in the size of the band, ceteris paribus, increases the costs of being away from the frictionless optimal level of the control variable but reduces the adjustment costs.

When information arrives continuously, the size of the band increases with the diffusion parameter because maintaining the size constant will imply a substantial increase in adjustment costs, while not changing much the costs of being away from the optimal level. The unavoidable increase in costs is minimized when the size of the band becomes wider and both costs are increased.

When there are infrequent information arrivals, something different happens. For a higher value of the diffusion parameter, if the size of the band is kept the same, there is a higher probability that the absolute value of the discrepancy reaches a higher level at times of no information. So, the expected costs of being away from the optimal level of the control variable increase substantially due to the convexity of the flow costs, even with the same barriers. This differs from the perfect information case where only adjustment costs increase when the barriers are kept constant. Therefore, in the infrequent information case, there is less need to rebalance the increase in costs through an increase in the barrier size since both types of costs increase when the uncertainty increases and the barriers are kept constant.

A third pattern emerges from the table: the infrequent information barriers tend to respond less to the change in the diffusion parameter when this parameter is relatively large or when the adjustment costs are small.

To understand this result, notice that the difference between the two cases accentuates when the adjustments in the continuous case tend to occur at intervals that are small compared to the interval between information arrivals. Adjustments tend to occur more often in the continuous case when 
the adjustment cost is smaller or when the variance is larger. Therefore, it is in those cases that the infrequent information barriers are less responsive to changes in the diffusion parameter. As illustrated in Table 1, for a high adjustment cost, the barriers become less responsive to changes in the uncertainty parameter when the it gets larger. For a small adjustment cost, the optimal barriers stay practically constant for all values of the diffusion parameter. ${ }^{12}$

Finally, it can be seen in the table that the size of the band seems to be relatively more sensitive to the adjustment cost in the infrequent information case. The optimal band size should equalize the adjustment cost to the benefit of adjusting now rather than continuing with discrepancy $s$. In the infrequent information case, this benefit is less sensitive to $s$, since adjustments are only partially state-dependent. A more substantial increase in $s$ is therefore necessary in order to make the benefit of adjusting from $s$ now (and then following the optimal policy) equal to a higher adjustment cost level.

\section{$3 \quad$ Aggregate Effects of Macroeconomic Shocks}

In this section we discuss the aggregate implications of the optimal microeconomic rules we derived in the previous section based on both infrequent information and kinked adjustment costs. We calculate the relative effects of cumulative aggregate shocks between times of information arrival and average them according to their relative likelihood. We then compare our model with models where only one kind of imperfection is present: a full information adjustment cost model and an infrequent information model with no adjustment costs.

\subsection{Average Effect of Shocks in the Model with Ad- justment Costs and Infrequent Information}

In this subsection we first develop expressions for the effect of cumulative aggregate shocks of different sizes and for the average of these effects in the model with adjustment costs and infrequent information. For expositional

\footnotetext{
${ }^{12}$ We say "practically" because when calculated with a higher level of precision, it can be noticed that the barriers move, albeit very little.
} 
clarity, we start by assuming that there are no idiosyncratic shocks, to subsequently extend our formulas to include them. The effects depend on the initial distribution of deviations. We will assume that this distribution is the ergodic one, for which we develop the appropriate expression in the next subsection. Finally, we report and analyze the results.

\subsubsection{Analytical expression for the average effect of shocks}

We follow Bertola and Caballero (1990) in defining the macroeconomic variable as the negative of the average deviation of the control variable from its frictionless optimal level, where the average is taken over all agents. This deviation is also referred to as the disequilibrium level of the control variable. If the control variable is price, if the aggregate component of the frictionless optimal price is the nominal quantity of money and if output depends positively on the amount of real money, our defined macroeconomic variable will be an increasing function of the level of output, as in most money effects applications of the literature on state-dependent rules.

Let $y$ be our macroeconomic variable and consider the variation of $y$ in one period. During that period, aggregate shocks and idiosyncratic shocks to individual frictionless optimal levels of the control variable accumulate, but no adjustment is made until the information is received. Then, adjustment is made or not, depending on the level of disequilibrium revealed. In this section we explore the effect of aggregate shocks when both the information about the aggregate shock and the information about the idiosyncratic shock are released simultaneously to all agents.

The change in $y$ in one period is given by:

$$
\begin{gathered}
\Delta y=\int\left(\Delta x_{i}^{*}\right) d i-\int\left(\Delta x_{i}\right) d i \\
=\int\left(w+e_{i}\right) d i-\int\left(\Delta x_{i}\right) d i \\
=w-p_{u} E_{u}\left|\Delta x_{i}\right|+p_{d} E_{d}\left|\Delta x_{i}\right|
\end{gathered}
$$

where $e_{i}$ is the accumulated idiosyncratic shock of agent $i$ in one period, $w$ the accumulated aggregate shock in one period, $p_{u}$ the fraction of upward adjustments, $p_{d}$ the fraction of downward adjustments, $E_{u}\left|\Delta x_{i}\right|$ the average size of the upward adjustments and $E_{d}\left|\Delta x_{i}\right|$ the average size of downward adjustments. We are assuming that agents differ only by the level of disequilibium and the idiosyncratic shocks they receive. We also assume that the number of individual agents is very large and that the idiosyncratic shocks 
have zero mean. As a consequence, the average among agents of idiosyncratic shocks is zero. The fraction of agents who adjust upwards is formed by agents that realize after information arrives that the frictionless optimal level of their control variable exceeds the actual level by more than $s$. Thus, each agent adjusts its control variable by at least $s$. A similar reasoning applies to the fraction of agents which adjusts downwards.

Effects of cumulative aggregate shocks with no idiosyncratic uncertainty To know the effect of a cumulative aggregate shock $w$ it is necessary to know both the fraction and the average size of upward and downward adjustments, which depend only on the distribution of the initial disequilibrium when there are no idiosyncratic shocks.

Whenever there is a positive accumulated aggregate shock, $w>0$, some units will adjust upwards if their discrepancy after the shock exceeds $s$, while others will not adjust because their discrepancy will remain within barriers. The size of the upward adjustment for each unit is always equal to the absolute value of the final disequilibrium, that is $|z-w|=-(z-w)$. This is represented in Figure 1. The effect is totally symmetric in the case of a negative aggregate shock. Thus, we have the following expression for the effect of a positive aggregate shock:

$$
\begin{gathered}
{[\Delta y \mid w>0]=w-F_{t}(-s+w) \int_{-s}^{-s+w} \frac{-(z-w)}{F_{t}(-s+w)} d F_{t}(z)} \\
=w-\int_{-s}^{-s+w}-(z-w) d F_{t}(z)
\end{gathered}
$$

It is useful to express the effect as a ratio to the shock. Then:

$$
\begin{aligned}
& {\left[\frac{\Delta y}{w} \mid w>0\right]=1+\int_{-s}^{-s+w}\left(\frac{z}{w}-1\right) d F_{t}(z)} \\
& \quad=\left[1-F_{t}(-s+w)\right]+\int_{-s}^{-s+w} \frac{z}{w} d F_{t}(z)
\end{aligned}
$$

The first expression between brackets is the proportion of units that do not adjust multiplied by the relative effect which would occur if they were the only units, that is one. The integral represents the average effect which would take place if the units which adjust were the only units, multiplied by their proportion. Thus the total relative effect is a weighted average between the full effect and the effect generated by the units that adjust. If $F_{t}$ is symmetric the latter effect is always negative. As $w$ increases from zero, the proportion of units that do not adjust decreases. Although the proportion of agents that do adjust increases, the average size of their adjustment decreases as a 
proportion to the shock size ${ }^{13}$. A small shock only triggers adjustments from units with large disequilibrium, so the size of adjustment is relatively large compared to the shock. Larger shocks will trigger adjustment from units with relatively small disequilibrium, so the average relative size of adjustments is reduced, although the proportion of units that adjust is increased. The prevalent effect when we increase $w$ is unclear. We know however what happens for the extreme values of $w$.

A close to zero aggregate shock will have full effect since almost no unit will adjust. This can be shown by taking the limit as $w$ goes to zero of expression (5). On the other hand, a shock larger than $2 s$ will have no effect if the initial distribution of price deviations is symmetric, since all units adjust with the average size of adjustments equal to $w$ (since by symmetry the average initial disequilibrium is zero). Analytically, the term between square brackets in (5) and the integral term become zero.

Observe that expression (5) is continuous in $w$ if $F_{t}$ is continuous. As we will see in the next section on the ergodic distribution, $F_{t}$ tends to be discontinuous in zero. However, this cannot cause a discontinuity in the effect since $d F_{t}$ is multiplying $z$ in the integral and $F_{t}$ jumps when $z$ is equal to zero. So the effect should decrease from one to zero when $w$ increases from zero to $2 s^{14}$.

If the shock is negative the relative effect is the same, as long as the distribution of deviations is symmetric.

We should observe that the cumulative effects of aggregate shocks with no idiosyncratic uncertainty in our framework is analogous to the effect of an instantaneous large aggregate shock in a model with continuous information. However, in the latter framework, the optimal rule is generated under the assumption that the path of the frictionless optimal level of the control variable is continuous. So a large instantaneous shock violates the assumptions of the model.

\section{Effects of cumulative aggregate shocks when idiosyncratic uncer-} tainty is present The existence of idiosyncratic shocks increases the fre-

\footnotetext{
${ }^{13}$ The reason is that the size of each unit's adjustment is equal to the size of the disequilibrium after the shock, and the latter is composed by the sum of the initial disequilibrium, $\mathrm{z}$, with the shock.

${ }^{14}$ For general $\mathrm{F}$ there is no guarantee however that it is strictly decreasing in the size of the shock.
} 
quency of both positive and negative adjustments at times of information arrival. Without further assumptions, we cannot tell if it will magnify or dampen the effect of aggregate shocks. If the distribution of price deviations is decreasing in the absolute size of the deviations, then the idiosyncratic uncertainty tends to attenuate the effect of aggregate shocks. This is because a positive aggregate shock, for example, tends to simultaneously make the left side of the distribution of price deviations thicker while leaving the right side empty. Then the added idiosyncratic shocks cause many more upward adjustments than dowward adjustments. As a result, the effect of a positive shock is dampened. Obviously, a symmetric version of the same mechanism works for negative shocks too.

In Appendix B we first derive an analytical expression for the average relative effect of a cumulative aggregate shock of a given size:

$$
\begin{aligned}
E\left[\frac{\Delta y}{w} \mid w\right] & =1-\frac{1}{w} \int_{-s}^{s}\left[\int_{z-w+s}^{\infty}-\left(\frac{z-w-e_{i}}{\sigma_{i}}\right) \phi\left(\frac{e_{i}}{\sigma_{i}}\right) d e_{i}\right. \\
& \left.-\int_{-\infty}^{z-w-s}\left(\frac{z-w-e_{i}}{\sigma_{i}}\right) \phi\left(\frac{e_{i}}{\sigma_{i}}\right) d e_{i}\right] d F(z)
\end{aligned}
$$

where $\mathrm{F}$ is the ergodic (average) distribution of price deviations (see the subsection below). Then these effects are averaged according to the likelihood of each shock size to yield an expression for the average relative effect of an unspecified aggregate shock:

$$
\begin{gathered}
E\left[\frac{\Delta y}{w}\right]=1-\int_{-\infty}^{\infty} \frac{1}{w \cdot \sigma_{A}} \phi\left(\frac{w}{\sigma_{A}}\right)\left\{\int _ { - s } ^ { s } \left[\int_{z-w+s}^{\infty}-\left(\frac{z-w-e_{i}}{\sigma_{i}}\right) \phi\left(\frac{e_{i}}{\sigma_{i}}\right) d e_{i}-\right.\right. \\
\left.\left.\int_{-\infty}^{z-w-s}\left(\frac{z-w-e_{i}}{\sigma_{i}}\right) \phi\left(\frac{e_{i}}{\sigma_{i}}\right) d e_{i}\right] d F(z)\right\} d w
\end{gathered}
$$

\subsubsection{The ergodic distribution of deviations}

Although there is no invariant distribution of deviations in the presence of aggregate shocks, the average distribution coincides with the ergodic distribution, i.e. the distribution that would remain invariant if all units only had idiosyncratic shocks. The ergodic distribution for this class of rule has an atom at zero, since many units adjust their discrepancy simultaneously to zero at the time information arrives. The conditions that determine the ergodic distribution are given in Appendix C. 
Despite the fact that aggregate shocks occur continuously and are always small in magnitude, adjustments are large, infrequent and have a large degree of simultaneity. Simultaneity results from the infrequent release of information about aggregate shocks, which makes the magnitude of news relatively large, even though innovations are small and occur continuously.

\subsubsection{Results}

Figure 2 shows the ergodic distribution corresponding to two different values of the diffusion parameter. We observe that the higher the diffusion parameter, the flatter the density curve and the higher the probability associated with the atom at zero. This is consistent with the fact that a higher variance triggers more adjustments at times of information arrival, giving more weight to the atom. Additionally, as there is more movement in the deviation between adjustment times, the density gets flatter. These results tell us that the higher the aggregate uncertainty, the greater the simultaneity of actions. Intuitively, with higher uncertainty, information arrivals bring about more news more often. A large piece of news, that is a large cumulative aggregate shock since the last information arrival, triggers simultaneous adjustments from a large number of agents.

Since the ergodic distributions are symmetric and decreasing in the absolute size of the price deviation, we can rely on the analysis of theeffect of cumulative aggregate shocks developed in section 3.1.1. Figure 3 shows that the relative effect of a shock is decreasing with the absolute size of the shock, as anticipated in the previous description about the effect of aggregate shocks. Table 2 shows the average effect of a shock for different total variances of shocks and different decompositions of this total variance between aggregate and idiosyncratic variances of shocks. The average effect decreases when we keep aggregate uncertainty constant and increase idiosyncratic uncertainty, as anticipated in section 3.1.1. When we keep idiosyncratic variance constant and increase aggregate variance, the average effect is also reduced ${ }^{15}$. This result follows solely from the higher likelihood of large shocks, which have a lower relative effect, since the aggregate uncertainty does not affect the impact of any specific aggregate shock. When we keep total uncertainty con-

\footnotetext{
${ }^{15}$ It should be noted that the effect is not reduced as much as one might have expected, but one has to realize that the band increases as the total uncertainty increases, thereby lowering the effect.
} 
stant but increase the relative weight of the idiosyncratic shocks, the effect is reduced indicating that the influence of idiosyncratic uncertainty on each specific shock is prevalent over the way the shocks are averaged. Finally, we observe that the size of adjustment costs has a very important influence on the results. This is because the size of the bands is very sensitive to adjustment costs. A reduction in adjustment costs reduces the size of the band substantially, decreasing the proportion of units that do not adjust and, as a consequence, the relative effect of an aggregate shock.

\subsection{Comparison with other models}

In this section we compare the features of aggregate effects in the model with infrequent information and lump-sum adjustment costs to the ones obtained in models where only one kind of imperfection is present.

\subsubsection{Adjustment cost with full information}

A lot of work has been done on the aggregate effects of shocks in adjustment cost models with full information, as referred to in the introduction. Caballero and Engel (1992) develop a method to quantify the average effect of aggregate shocks and apply it to assess output effects. However, their method measures instantaneous effects, and our objective is to compare aggregate effects in both infrequent information and full information models during the same time horizon ${ }^{16}$. The convenient time horizon we choose is the time between information arrivals.

We want to evaluate the effect generated by cumulative aggregate shocks of different sizes. First, it should be noticed that two cumulative aggregate shocks of the same size may have different effects even if the initial distribution of price deviations is the same. This is due to the hysteresis built in the models: the path of aggregate shocks matter, not just their cumulative sum. Given this feature, to obtain a unique measure of the relative effect of a cumulative aggregate shock of a given size, we have to average the effect

\footnotetext{
${ }^{16}$ The relative instantaneous average effect of an aggregate shock in a full information adjustment cost model with idiosyncratic uncertainty is always one. This follows because the ergodic distribution has density zero at the trigger points. The instantaneous effect in the infrequent information model is also one.
} 
of each shock of a given size with different components by the relative likelihood of its components. We therefore discretize the time and state space and perform Monte Carlo simulations of aggregate shocks, drawing paths according to their likelihood following the methodology in Bertola and Caballero (1990), and calculate the relative aggregate effect for each path. We then classify the cumulative shock sizes in small intervals and average the effects of cumulative shocks in each class, in order to obtain a representative average effect for each category of shock size. We also calculate a global average of all simulations.

In Figure 4 we graph our simulations according to each class of size shock. We notice that, in contrast with the infrequent information case in Figure 3 , the relative effect of shocks tends to stay constant, except for shocks that are very small. Average results are shown in Table 2. Average effects tend to be of similar magnitude but do not always go in the same direction. A higher aggregate uncertainty, all other parameters being kept constant, tends to increase the average effect, contrary to what we found in the infrequent information model.

\subsubsection{Infrequent information with no adjustment costs}

Caballero (1989) has worked out a model with no adjustment costs where part of the information relevant to each firm arrives at infrequent intervals, either through the payment of an information gathering cost or by observing the action of another firm which just paid its information gathering cost. There are some aggregate effects that come from sluggish adjustment to innovations in the frictionless optimal level of the control variable due to infrequent information. However, these effects do not last more than the time interval between information collections.

A nested simple version of our model with no adjustment cost would entail full adjustment every period of information arrival. Any shock will have full effect until the time of information arrival, when the effect will be eliminated by the full adjustment of all units. Therefore, if we use the same time interval we used above to measure the effect of a cumulative aggregate shock in the models with adjustment costs, we find no aggregate effect. 


\section{Final Comments and Extensions}

The need to put information gathering costs and adjustment costs together to yield optimal rules that are both time and state dependent has long been recognized by researchers in the macroeconomic literature (see, for example, Blanchard and Fischer (1989), p.413 and Caballero (1989), p.29). This paper makes an important step forward in this direction by assuming infrequent information about the optimal control variable and lump-sum adjustment costs, and deriving resulting optimal rules that are both time and state dependent. From this point it does not seem difficult to endogenize information arrival by introducing costs of information collection. However, as argued in the introduction, infrequent exogenous information arrival is realistic per se in various contexts. A more difficult task is to generalize the current model to stochastic processes that are not martingales: then there may be adjustments between intervals of information collection, and inertia bands in this interval should depend on the remaining time before the next information arrival. Also, the same difficulties would appear if we extended the model to allow part of the stochastic component to be continuously observed.

In the aggregation of our simple rules we used a specific assumption: all agents receive information at the same time. This assumption, although extreme, captures a realistic feature of the economic world: some important information such as the release of macroeconomic statistics, tends to reach a lot of economic agents at the same time. Using this assumption, we arrived at the result that a higher aggregate uncertainty increases the simultaneity of agents' actions. Other distinctive results are that the effect of cumulative aggregate shocks tends to decrease with the absolute size of the aggregate shock, and that the larger the aggregate uncertainty, the lower the average effect of shocks. We also find implications which are shared with models of adjustment cost with full information: a higher idiosyncratic uncertainty and a lower adjustment cost both tend to reduce the effect of an aggregate shock. The aggregation part could be extended to include heterogeneity in information arrival times and information externality among agents, as in Caballero (1989). However, given the differences between information structures that are appropriate for various areas of macroeconomics where adjustment costs apply, we leave these extensions for specific applications of the model. 


\section{References}

Ball, L. and S. Cecchetti (1988), "Imperfect Information and Staggered Price Setting," American Economic Review 78: 999-1018.

Ball, L. and G. Mankiw (1994), "Asymmetric Price Adjustments and Economic Fluctuations", Economic Journal 104: 247-261.

Blanchard, O. and S. Fischer (1980), "Lectures on Macroeconomics,"MIT Press.

Banerjee, O. (1992), "A Simple Model of Herd Behavior, " Quarterly Journal of Economics 107: 797-817.

Bertola, G. and R. Caballero (1990), "Kinked Adjustment Costs and Aggregate Dynamics, " in NBER Macroeconomics Annual 1990, O. Blanchard and S. Fischer (eds.), Cambridge, MA: The MIT Press 1990.

Bonomo, M. (1994), "Optimal Two-sided and Suboptimal One-sided Pricing Rules", Working Paper no. , Pontificia Universidade Catolica do Rio de Janeiro (PUC-Rio).

Caballero, R. (1989), "Time Dependent Rules, Aggregate Stickiness and Information Externalities, " Working Paper no. 428, Columbia University.

Caballero, R. (1993), "Durable Goods: An Explanation for their Slow Adjustment," Journal of Political Economy 101: 351-384.

Caballero, R. and E. Engel (1992), "Price Rigidities, Asymmetries and Output Fluctuations, " NBER Working Paper no. 4091.

Caballero, R. and E. Engel (1993), "Heterogeneity and Output Fluctuations in a Dynamic Menu-Cost Economy," Review of Economic Studies 60: 95-119.

Caballero, R. and E. Engel (1994), "Explaining Investment Dynamics in U.S. Manufacturing: A Generalized (S,s) Approach," mimeo

Caballero, R., Engel, E. and J. Haltiwanger (1995), " Aggregate Employment Dynamics: Building from Microeconomic Evidence," mimeo.

Caplin, A. (1985), "The Variability of Aggregate Demand with (S,s) Inventory Policies," Econometrica 53: 1395-1410.

Caplin, A. and J. Leahy (1991), "State Dependent Pricing and the Dynamics of Money and Output," Quarterly Journal of Economics 106: 683708.

Caplin, A. and D. Spulber, "Menu Costs and the Neutrality of Money," Quarterly Journal of Economics 102: 703-726. 
Caplin, A. and J. Leahy (1994), "Business as Usual, Market Crashes, and Wisdom After the Fact ," American Economic Review 84: 548-565.

Cornell, B. (1978), "Using the Options Pricing Model to Measure the Uncertainty Producing Effect of Major Announcements," Financial Management 7, 54-59.

Dixit, A. (1993), "The Art of Smooth Pasting," Fundamentals of Pure and Applied Economics series. Harwood Academic Publishers.

Ederington, L.H. and J. H. Lee (1993), "How Markets Process Information: News Releases and Volatility," Journal of Finance 48: 1161-1192.

Harvey, C.R. and R.D. Huang (1992), "Information Trading and Fixed Income Volatility," mimeo.

Sargent, T. (1987), "Dynamic Macroeconomic Theory," Harvard University Press. 


\section{Appendix A}

The function B represents the expected discounted cost of departing from the frictionless optimal level of the control variable between now and the time of the next information arrival. It is derived as follows

$$
\begin{gathered}
B(y)=E_{t}\left(\int_{0}^{1} e^{-\rho z}\left(x_{t+z}-x_{t+z}^{*}\right)^{2} d z\right) \\
=E_{t}\left(\int_{0}^{1} e^{-\rho z}\left(y+\sigma\left(w_{t+z}-w_{t}\right)\right)^{2} d z\right) \\
=\int_{0}^{1} e^{-\rho z} E_{t}\left(y+\sigma\left(w_{t+z}-w_{t}\right)\right)^{2} d z \\
=\int_{0}^{1} e^{-\rho z}\left(y^{2}+\sigma^{2} E_{t}\left(w_{t+z}-w_{t}\right)^{2}\right) d z \\
\quad=\int_{0}^{1} e^{-\rho z} y^{2} d z+\int_{0}^{1} e^{-\rho z} \sigma^{2} z d z \\
\quad=\frac{y^{2}\left(1-e^{-\rho}\right)}{\rho}-\frac{\sigma^{2} e^{-\rho}}{\rho}+\frac{\sigma^{2}\left(1-e^{-\rho}\right)}{\rho^{2}}
\end{gathered}
$$

In the second equality, we decompose the discrepancy in $t+z$ into the sum of the discrepancy in $t$, which is $y$, and the change in $x^{*}$ between $t$ and $t+z$. The third equality results from applying Fubini's theorem, while the two next ones use respectively the conditional independence of increments of the Wiener process and the formula for their variance. The last equality is obtained by calculating the integrals, the second one with an integration by parts.

\section{Appendix B}

We develop below analytical expressions to quantify the effects of cumulative aggregate shocks in the presence of aggregate uncertainty.

Our general formula (4) in the text tells us that to evaluate the effect of a cumulative aggregate shock $w$, it is necessary to know both the fraction and the average size of upward and downward adjustments. Both depend on the initial distribution of agents' deviations and on the cumulative idiosyncratic shock which affected the optimal level of the control variable for each agent. Since realizations of the idiosyncratic shocks across the economy are generally unknown, we evaluate the average effect of a known aggregate shock $w$, by averaging over all possible realizations of the $e_{i}$ shocks weighted by their likelihood. As a first step, suppose that all agents have the same initial 
discrepancy $z$. Then the effect of an aggregate shock $w$ will be:

$$
\begin{aligned}
E[\Delta y \mid w, z]=w & -\left(1-\Phi\left(\frac{z-w+s}{\sigma_{i}}\right)\right) \int_{z-w+s}^{\infty}-\left(z-w-e_{i}\right) \frac{\phi\left(\frac{e_{i}}{\sigma_{i}}\right)}{\sigma_{i}\left(1-\Phi\left(\frac{z-w+s}{\sigma_{i}}\right)\right)} d e_{i} \\
& +\Phi\left(\frac{z-w-s}{\sigma_{i}}\right) \int_{-\infty}^{z-w-s}\left(z-w-e_{i}\right) \frac{\phi\left(\frac{e_{i}}{\sigma_{i}}\right)}{\sigma_{i} \Phi\left(\frac{z-w-s}{\sigma_{i}}\right)} d e_{i}
\end{aligned}
$$

The term between parentheses multiplying the first integral is the probability that a discrepancy of level $z$, after accounting for the known aggregate shock $w$ and the normally distributed idiosyncratic shocks, becomes smaller than $-s$, triggering an upward adjustment. Thus, the first integral is the expected size of the upward adjustment, conditioned on the occurence of such an adjustment. The second integral and the term that multiplies it apply to downward adjustments and have similar interpretations.

The initial discrepancies of the units at $t$, rather than being concentrated on a specific value of $z$, are distributed according to some distribution $F_{t}$. Assuming that there are many units at each position $z$, such that the frequency of idiosyncratic shocks for all units at a given position can be well approximated by its probability distribution, we can average the effect of an aggregate shock, as calculated above for a given $z$, according to the distribution $F_{t}$ of the $z^{\prime} s$. Then,

$E\left[\Delta y \mid w, F_{t}\right]=w-\int_{-s}^{s}\left[\int_{z-w+s}^{\infty}-\left(\frac{z-w-e_{i}}{\sigma_{i}}\right) \phi\left(\frac{e_{i}}{\sigma_{i}}\right) d e_{i}-\int_{-\infty}^{z-w-s}\left(\frac{z-w-e_{i}}{\sigma_{i}}\right) \phi\left(\frac{e_{i}}{\sigma_{i}}\right) d e_{i}\right] d F_{t}(z)$

Next, we express the effect as a ratio, dividing the above expression by $w$, resulting in:

$$
\begin{gathered}
E\left[\frac{\Delta y}{w} \mid w, F_{t}\right]=1-\frac{1}{w} \int_{-s}^{s}\left[\int_{z-w+s}^{\infty}-\left(\frac{z-w-e_{i}}{\sigma_{i}}\right) \phi\left(\frac{e_{i}}{\sigma_{i}}\right) d e_{i}\right. \\
\left.-\int_{-\infty}^{z-w-s}\left(\frac{z-w-e_{i}}{\sigma_{i}}\right) \phi\left(\frac{e_{i}}{\sigma_{i}}\right) d e_{i}\right] d F_{t}(z)
\end{gathered}
$$


Now we take the expectation with respect to $w$, yielding:

$$
\begin{aligned}
& E\left[\frac{\Delta y}{w} \mid F_{t}\right]=1-\int_{-\infty}^{\infty} \frac{1}{w \cdot \sigma_{A}} \phi\left(\frac{w}{\sigma_{A}}\right)\left\{\int _ { - s } ^ { s } \left[\int_{z-w+s}^{\infty}-\left(\frac{z-w-e_{i}}{\sigma_{i}}\right) \phi\left(\frac{e_{i}}{\sigma_{i}}\right) d e_{i}-\right.\right. \\
& \left.\left.\int_{-\infty}^{z-w-s}\left(\frac{z-w-e_{i}}{\sigma_{i}}\right) \phi\left(\frac{e_{i}}{\sigma_{i}}\right) d e_{i}\right] d F_{t}(z)\right\} d w
\end{aligned}
$$

The expression above evaluates the average effect of a shock for a given initial distribution of deviations. Finally, taking expectations with respect to the distribution of deviations (and using Fubini's theorem), we arrive at:

$$
\begin{gathered}
E\left[\frac{\Delta y}{w}\right]=1-\int_{-\infty}^{\infty} \frac{1}{w \cdot \sigma_{A}} \phi\left(\frac{w}{\sigma_{A}}\right)\left\{\int _ { - s } ^ { s } \left[\int_{z-w+s}^{\infty}-\left(\frac{z-w-e_{i}}{\sigma_{i}}\right) \phi\left(\frac{e_{i}}{\sigma_{i}}\right) d e_{i}-\right.\right. \\
\left.\left.\int_{-\infty}^{z-w-s}\left(\frac{z-w-e_{i}}{\sigma_{i}}\right) \phi\left(\frac{e_{i}}{\sigma_{i}}\right) d e_{i}\right] d F(z)\right\} d w
\end{gathered}
$$

where $F$ is the ergodic (average) distribution of deviations.

\section{Appendix $C$}

In this appendix we derive the equations which determine the ergodic distribution.

Let $f_{t}($.$) be the density function for price deviations different from zero$ at time $\mathrm{t}$, immediately after information arrival and adjustments are made. Let $P_{t}(0)$ be the fraction of units with price deviation zero at time $\mathrm{t}$, after the adjustments are made. Let $v_{i}$ be the total cumulative shock to the frictionless optimal level of the control variable of unit $i$ during the period of time without information. So, $v_{i}=w+e_{i}$ and $v_{i}$ is normally distributed with zero mean and variance $\sigma^{2}=\sigma_{A}^{2}+\sigma_{I}^{2}$.

It is clear that $f_{t+1}(z)=0$ for $z<-s$ or $z>s$. For $-s<z<s$, and $z \neq 0, f_{t+1}$ is given by:

$$
f_{t+1}(z)=\int_{-z-s}^{-z+s} f_{t}(z+v) \frac{1}{\sigma} \phi\left(\frac{v}{\sigma}\right) d v+P_{t}(0) \phi\left(\frac{-z}{\sigma}\right)
$$


The fraction of units at zero in $t+1$ relates to the distribution in $t$ in the following way:

$$
P_{t+1}(0)=\int_{-s}^{s} f_{t}(z)\left[\Phi\left(\frac{z-s}{\sigma}\right)+1-\Phi\left(\frac{z+s}{\sigma}\right)\right] d z+P_{t}(0) \phi(0)
$$

For the distribution to be well defined it has to satisfy for all $t$ :

$$
P_{t}(0)+\int_{-s}^{s} f_{t}(z) d z=1
$$

Making $P_{t+1}=P_{t}$ and $f_{t+1}=f_{t}$ in the conditions above determines the ergodic distribution. Notice that any two of the three conditions above imply that the third one is satisfied. Only two conditions are therefore necessary to determine the ergodic distribution. 
Figure 1 - Effect of a Positive Aggregate Shock $w$ (with no idiosyncratic uncertainty)

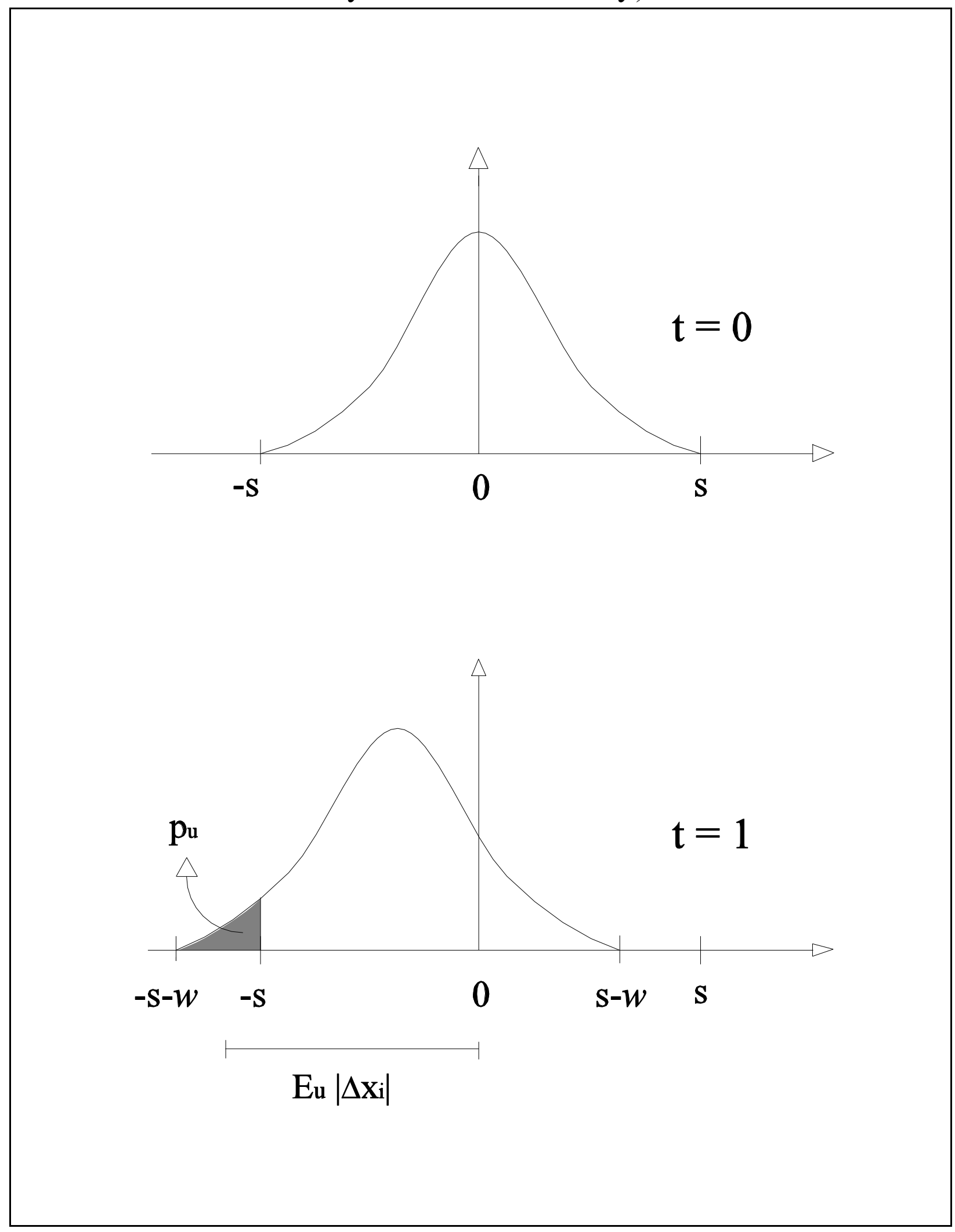


Figure 2 - Ergodic Distributions

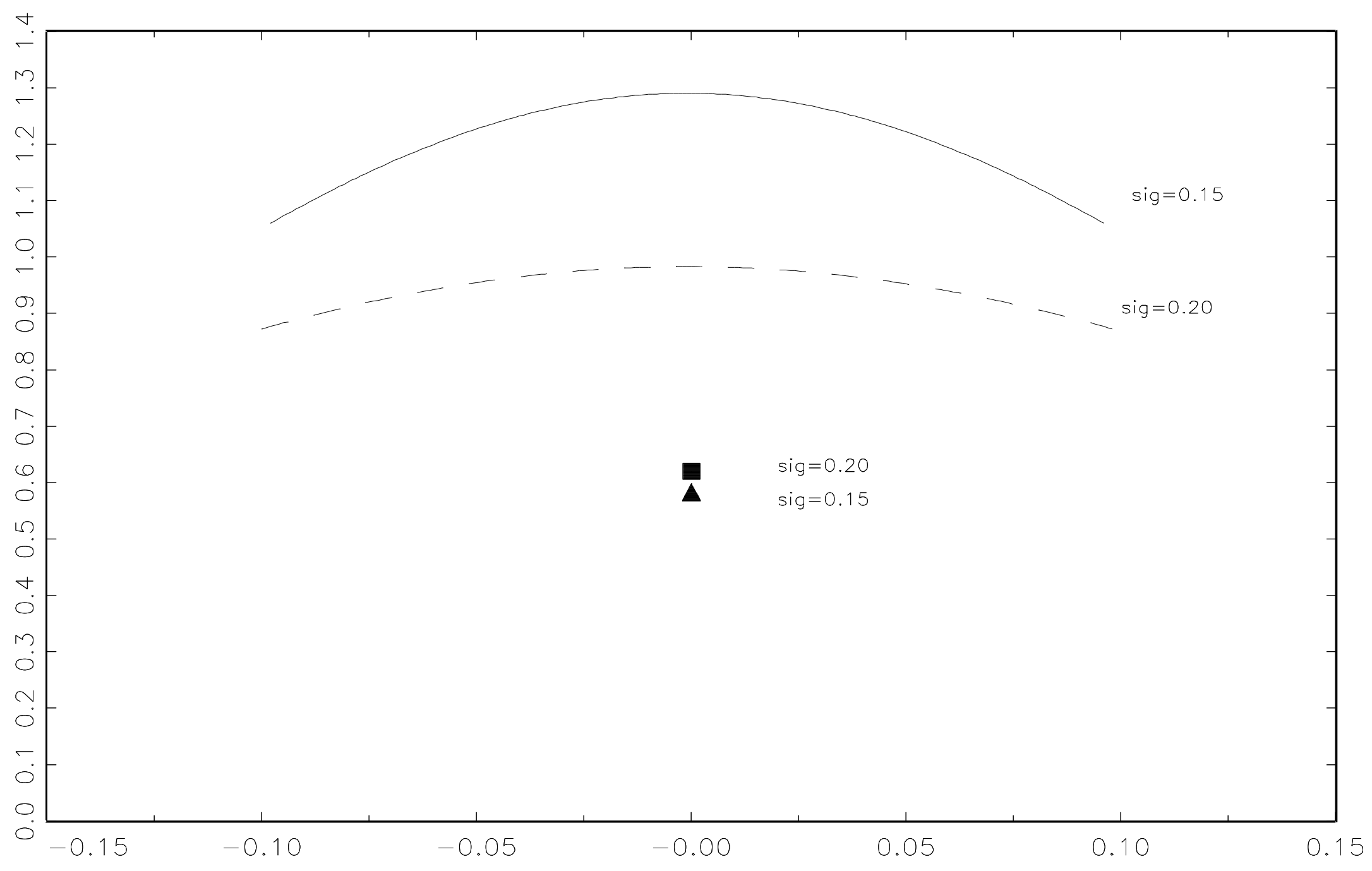


Figure 3 - Average Effect of Shocks Per Size of Shock $s=0.050, s a=0.043, s i=0.025, k=0.010, s=0.0810$

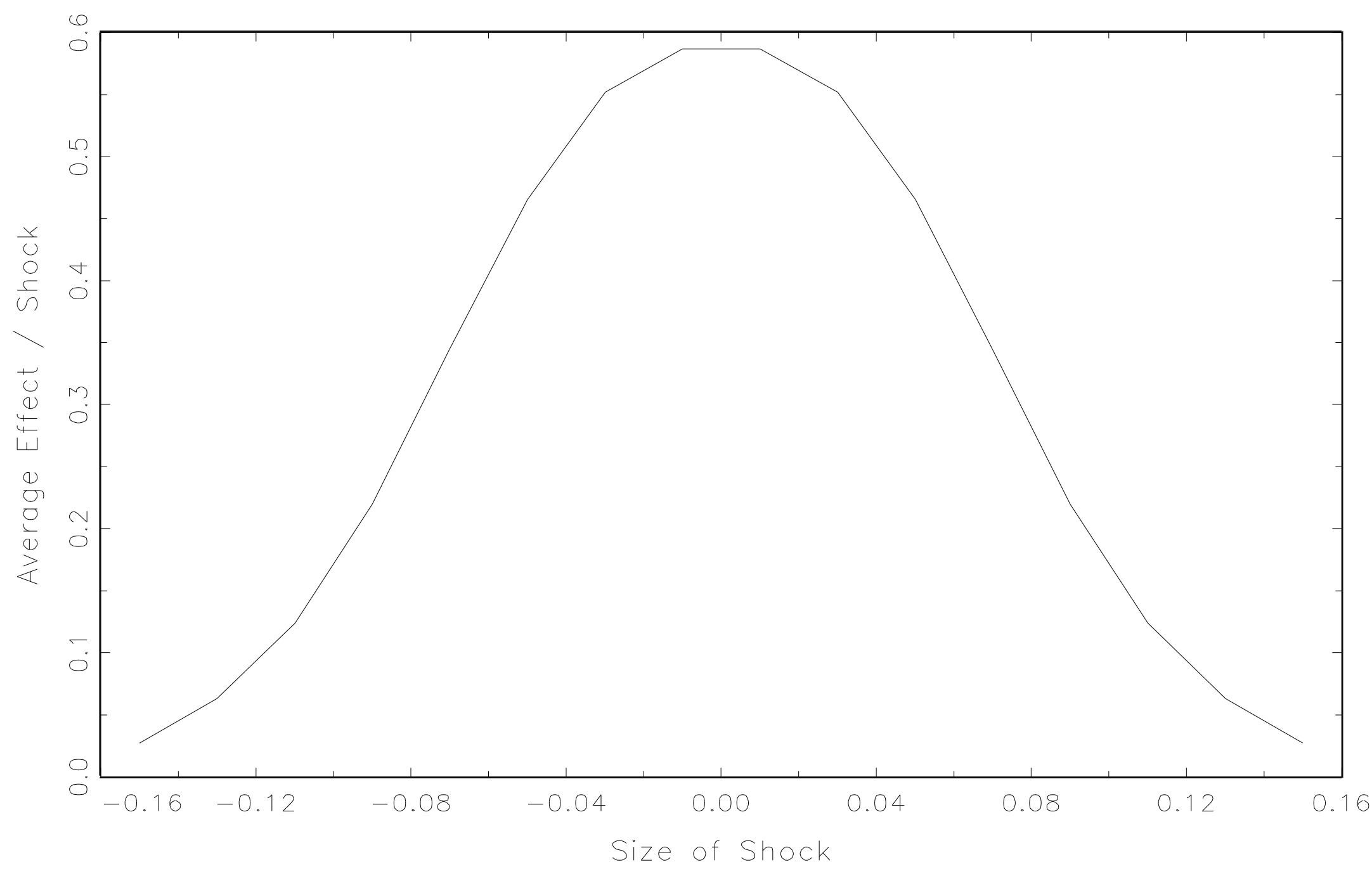


Figure 4 - Average Effect of Shocks Per Size of Shock

$s=0.100, s a=0.043, s i=0.090, k=0.010, s=0.1570$

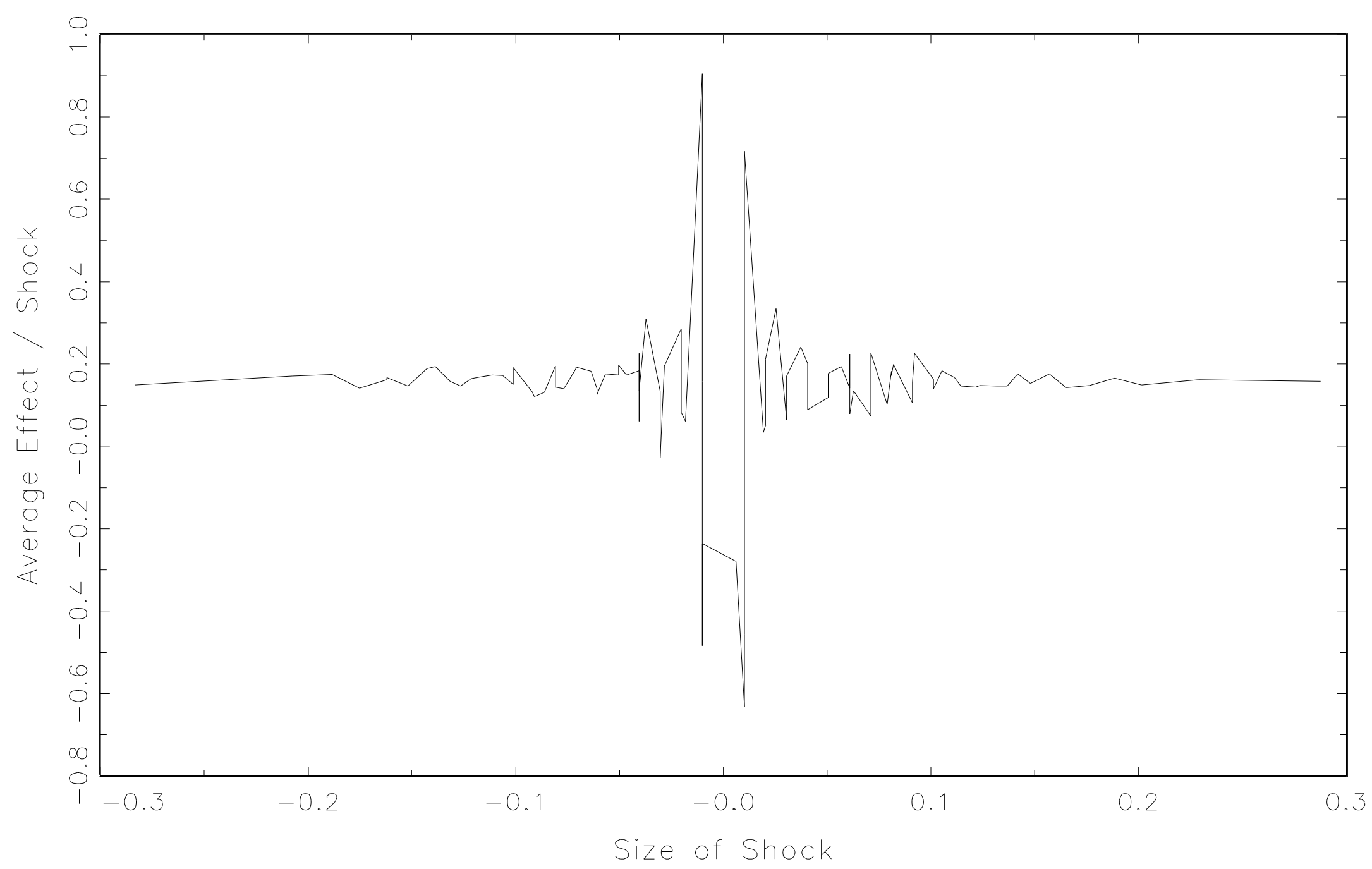

